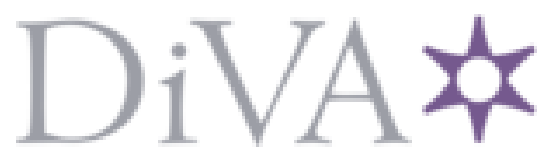

http://www.diva-portal.org

This is the published version of a paper presented at 2021 IEEE International Symposium on Information Theory (ISIT).

Citation for the original published paper:

Zhou, L., Oechtering, T J., Skoglund, M. (2021)

Incremental Design of Secure Biometric Identification and Authentication

In:

https://doi.org/10.1109/ISIT45174.2021.9518285

N.B. When citing this work, cite the original published paper.

Permanent link to this version:

http://urn.kb.se/resolve?urn=urn:nbn:se:kth:diva-301142 


\title{
Incremental Design of Secure Biometric Identification and Authentication
}

\author{
Linghui Zhou, Student Member, IEEE, Tobias J. Oechtering, Senior Member, IEEE, \\ and Mikael Skoglund, Fellow, IEEE.
}

\begin{abstract}
In this work, we study incrementally designed secure biometric identification and authentication systems, in which a new system is designed based on an existing legacy system. By taking privacy and secrecy into consideration, we characterize the fundamental trade-offs among the identification rate, secret key rates, and privacy leakage rate.
\end{abstract}

\section{INTRODUCTION}

With advances in smart technologies, biometrics are widely used in authentication and identification systems, which usually involve public helper data [1], [2]. Nowadays, the biometric information of a user is usually used in multiple systems. Hence, it is motivated to study efficient design. In some scenarios, there is already an existing legacy system and a new system is built based on the legacy system, which is the incremental design considered in this work. By reusing the legacy system, a new system can be implemented more sustainably by building on the legacy system. Additionally, information can be leaked about the biometrics and the secret keys from the public helper data. This motivates characterizing the privacy and secrecy aspects among multiple biometric systems to enhance the preservation of privacy and secrecy.

The capacity of biometric identification systems is firstly characterized in [3]. The trade-off between the identification capacity and the storage is studied in [4]. By considering the privacy and secrecy aspects, biometric identification systems that allow authentication are studied in [2], [5], where the identification-secrecy-privacy tradeoff is studied. Multiple-use of biometric information in biometric authentication systems is considered in [6] to improve secrecy and efficiency. Fundamental limits on the performance of biometric identification and authentication with privacy and secrecy constraints are unknown if the biometric source is used repeatedly.

In this work, we study the incremental design of secure biometric identification systems that support authentication, which aims at designing a new system based on a legacy system. We want to maximize the identification rate to identify as many users as possible. We also want to maximize the secret key rates such that the secret keys are as long as possible and thus the system is more secure. To protect privacy

The work was partially supported by the Swedish Research Council under grant 2016-03853, the Digital Futures research center, and the Strategic Research Agenda Program, Information and Communication Technology The Next Generation (SRA ICT - TNG), through the Swedish Government.

Linghui Zhou, Tobias J. Oechtering, and Mikael Skoglund are with the Division of Information Science and Engineering, KTH Royal Institute of Technology and KTH Digital Future Center, 10044 Stockholm, Sweden (email: linghui@kth.se; oech@kth.se; skoglund@kth.se). better, we want to minimize the privacy leakage rate with respect to two public databases, which relates to the amount of information leaked about the biometric source sequence from the helper data in two helper databases. Taking the above considerations into account, the fundamental trade-offs among the identification rate, the secret key rates, and the privacy leakage rate are characterized.

\section{Problem Formulation}

We assume that the two systems enroll the same $M_{I}$ users. The model of a incrementally designed biometric identification and authentication system is depicted in Fig. 1, in which the new system is built based on the legacy system. The new overall design is however allowed to update the codebook and enrollments of the legacy system. Let $x^{N}(w)$ denote the biometric sequence of user $w$ in the enrollment phase, which is assumed to be identically independently distributed (i.i.d.) according to the probability mass function (p.m.f.) $Q_{X}(\cdot)$ defined on the finite alphabet $\mathcal{X}$. For each user $w \in\left[1: M_{I}\right]$, given the biometric sequence $x^{N}(w)$, the enrollment mapping $f_{i}(\cdot)$ of system $i \in\{1,2\}$ maps $x^{N}(w)$ to a secret key $s_{i}(w) \in\left[1: M_{S}^{i}\right]$ and helper data $j_{i}(w) \in\left[1: M_{J}^{i}\right]$, i.e.,

$$
f_{i}: x^{N}(w) \mapsto\left(s^{i}(w), j^{i}(w)\right) .
$$

The secret keys are stored in a secure database and later used for authentication. Helper data $\left\{j_{1}(w)\right\}_{w=1}^{M_{I}}$ of the legacy system and $\left\{j_{2}(w)\right\}_{w=1}^{M_{I}}$ of the new system, which are denoted with $\boldsymbol{J}_{1}$ and $\boldsymbol{J}_{2}$ in Fig. 1, are stored in two public databases. Note that we allow the new system to update the data and keys of the legacy system.

In the identification phase, an unknown but previously enrolled user $w$ is observed via a noisy memoryless channel $Q_{Y \mid X}$ and let $y^{N}$ denote the observation. The observed user index $w$ is assumed to be a realization of the random variable $W$ that is uniformly distributed on $\left[1: M_{I}\right]$. The identification mapping $g_{i}(\cdot)$ of system $i \in\{1,2\}$ uses $y^{N}$ and $\boldsymbol{J}^{i}$ to guess the user index as $\hat{w}$ and estimate the secret key as $\hat{s}_{i}$, i.e.,

$$
g_{i}:\left(y^{N}, \boldsymbol{J}^{i}\right) \mapsto\left(\hat{w}, \hat{s}_{i}\right),
$$

where $\boldsymbol{J}^{1}=\boldsymbol{J}_{1}$ and $\boldsymbol{J}^{2}=\left(\boldsymbol{J}_{1}, \boldsymbol{J}_{2}\right)$. If the estimated secret key matches the true secret key of the guessed user, i.e., $\hat{s}_{i}=$ $s_{i}(\hat{w})$, the user is granted access to the system $i$.

Definition 1: In an incrementally designed biometric identification and authentication system, a rate tuple $\left(R_{I}, R_{L}, R_{S}^{1}, R_{S}^{2}\right) \in \mathbb{R}_{+}^{4}$ of the identification rate, the privacy leakage rate, the secret key rates for the legacy system and 


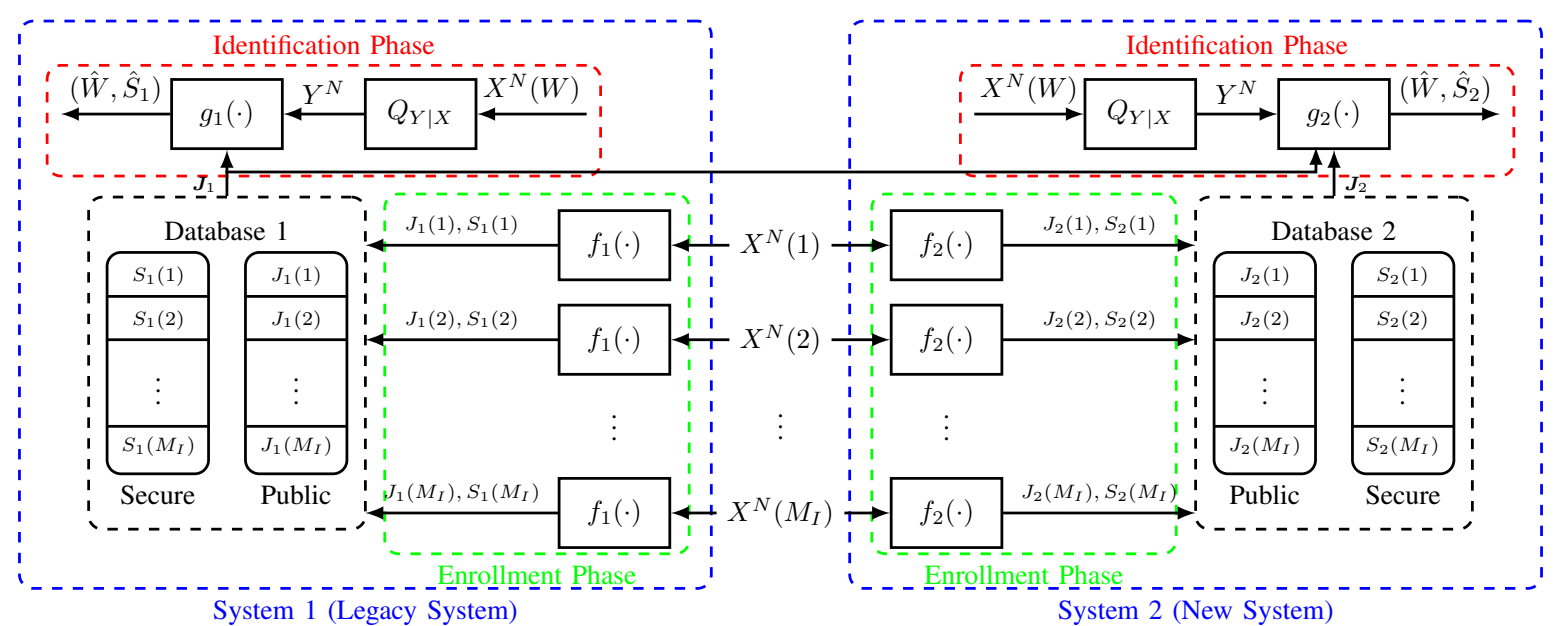

Fig. 1: Model of an incrementally designed biometric identification and authentication system.

the new system is achievable if, given any $\delta>0$ there exists some $N_{0}(\delta) \geq 1$, enrollment mapping $f_{i}(\cdot)$, and identification mapping $g_{i}(\cdot)$ such that for any $N \geq N_{0}(\delta)$ and $i \in\{1,2\}$, the following conditions are satisfied

$$
\begin{aligned}
\operatorname{Pr}\left\{\left(\hat{W}, \hat{S}_{i}\right) \neq\left(W, S_{i}(W)\right)\right\} & \leq \delta, \\
\log M_{I} & \geq N\left(R_{I}-\delta\right), \\
H\left(S_{i}(W)\right)+N \delta \geq \log M_{S}^{i} & \geq N\left(R_{S}^{i}-\delta\right), \\
I\left(X^{N}(W) ; J_{1}(W), J_{2}(W)\right) & \leq N\left(R_{L}+\delta\right), \\
I\left(S_{1}(W), S_{2}(W) ; J_{1}(W), J_{2}(W)\right) & \leq N \delta, \\
I\left(S_{1}(W) ; S_{2}(W)\right) & \leq N \delta .
\end{aligned}
$$

\section{MAin RESUlts}

We firstly review the fundamental limits of the singlesystem case [2] among the identification rate $R_{I}$, the privacy leakage rate $R_{L}$, and the secret key rate $R_{S}$ as follows

$$
\begin{aligned}
\mathcal{R}_{\mathrm{bi}}=\{ & \left(R_{I}, R_{L}, R_{S}\right) \in \mathbb{R}_{+}^{3}: R_{I}+R_{S} \leq I(U ; Y), \\
& R_{L} \geq I(U ; X \mid Y)+R_{I}, \\
& \text { for some } \left.P_{U X Y}=Q_{X} Q_{Y \mid X} P_{U \mid X}\right\}
\end{aligned}
$$

The following theorem characterizes the capacity region of the incrementally designed biometric identification and authentication system.

Theorem 1: Let $\mathcal{R}$ be a set of rate tuples $\left(R_{I}, R_{L}, R_{S}^{1}, R_{S}^{2}\right)$ as follows

$$
\begin{aligned}
\mathcal{R}=\{ & \left(R_{I}, R_{L}, R_{S}^{1}, R_{S}^{2}\right) \in \mathbb{R}_{+}^{4}: R_{I}+R_{S}^{1} \leq I\left(U_{1} ; Y\right), \\
& R_{I}+R_{S}^{1}+R_{S}^{2} \leq I\left(U_{2} ; Y\right), \\
& R_{L} \geq I\left(U_{2} ; X \mid Y\right)+R_{I}, \\
& \text { for some } \left.P_{U_{1} U_{2} X Y}=Q_{X} Q_{Y \mid X} P_{U_{2} \mid X} P_{U_{1} \mid U_{2}}\right\}
\end{aligned}
$$

Then a rate tuple $\left(R_{I}, R_{L}, R_{S}^{1}, R_{S}^{2}\right)$ is achievable in incrementally designed biometric identification and authentication systems if and only if $\left(R_{I}, R_{L}, R_{S}^{1}, R_{S}^{2}\right) \in \mathcal{R}$.

The p.m.f. given in (5d) is equivalent to the Markov chain $Y-X-U_{2}-U_{1}$. Hence a new system can be designed based on a legacy system if and only if this Markov chain condition holds. The incremental design of biometric systems in this work and the Markov chain condition can be related to the successive refinement of information [7], which aims at finding a finer description given a coarse description of the source. The process of finding a finer description is similar to the procedure of extracting new secret keys and helper data from the biometric sequence in the new system. It was shown in [7] that a rate-distortion problem is successively refinable if and only if the individual solution can be written as a Markov chain, which is similar to the Markov chain condition in $\mathcal{R}$. Further, $\mathcal{R}$ can be reduced to $\mathcal{R}_{\text {bi }}$ by letting $U_{2}=U_{1}$, which shows that the single-system case is a special case of the incremental design.

\section{Proof of Theorem 1}

\section{A. Achievability}

Fix p.m.f. $P_{U_{1} U_{2} X Y}=Q_{X} Q_{Y \mid X} P_{U_{2} \mid X} P_{U_{1} \mid U_{2}}$ and small enough $\epsilon, \delta>0$. Let $M_{I}=2^{N R_{I}}$ denote the number of users.

1) Legacy System:

Codebook generation: For fixed $\delta$, pick a rate pair $\left(R_{I}, R_{S}^{1}\right)$ and an auxiliary rate pair $\left(\tilde{R}_{S}^{1}, R_{J}^{1}\right)$ such that

$$
\begin{aligned}
& R_{S}^{1} \leq I\left(U_{1} ; Y\right)-2 \delta-R_{I}, \\
& \tilde{R}_{S}^{1}=I\left(U_{1} ; Y\right)-2 \delta-R_{I}-R_{S}^{1}, \\
& R_{J}^{1}=I\left(U_{1} ; X \mid Y\right)+R_{I}+3 \delta .
\end{aligned}
$$

Randomly and independently generate $2^{N\left(I\left(U_{1} ; X\right)+\delta\right)}$ i.i.d. codewords $u_{1}^{N}\left(j_{1}, s_{1}, \tilde{s}_{1}\right)$ according to $\prod_{i=1}^{N} P_{U_{1}}\left(u_{1, i}\right)$. We distribute the codewords uniformly at random into $2^{N R_{J}^{1}}$ bins indexed by $j_{1}$, and each bin consists of $2^{N\left(R_{S}^{1}+\tilde{R}_{S}^{1}\right)}$ codewords. We further distribute the codewords in each bin into $2^{N R_{S}^{1}}$ subbins indexed by $s_{1}$ and each subbin consists of $2^{N \tilde{R}_{S}^{1}}$ codewords indexed by $\tilde{s}_{1}$.

Enrollment: For each user $w$, we look for a codeword index triple $\left(j_{1}(w), s_{1}(w), \tilde{s}_{1}(w)\right)$ such that $\left(u_{1}^{N}\left(j_{1}(w), s_{1}(w), \tilde{s}_{1}(w)\right), x^{N}(w)\right) \in \mathcal{T}_{\epsilon}^{N}$. If no such index triple $\left(j_{1}(w), s_{1}(w), \tilde{s}_{1}(w)\right)$ exists, an index triple $\left(j_{1}(w), s_{1}(w), \tilde{s}_{1}(w)\right)$ is randomly drawn from $\left[1: 2^{N R_{J}^{1}}\right] \times$ $\left[1: 2^{N R_{S}^{1}}\right] \times\left[1: 2^{N R_{S}^{1}}\right]$. If there are more than one such index triple $\left(j_{1}(w), s_{1}(w), \tilde{s}_{1}(w)\right)$, one of them is selected uniformly 
at random. The helper data and the secret key of user $w$ is generated as $j_{1}(w)$ and $s_{1}(w)$, respectively. The codebooks and mappings are revealed to all parties.

Identification and Authentication: After user $w$ is observed, the observation $y^{N}$ is provided to the system. It checks all the entries in the database and looks for a triple $\left(\hat{w}, \hat{s}_{1}, \tilde{s}_{1}\right)$ such that $\left(u_{1}^{N}\left(j_{1}(\hat{w}), \hat{s}_{1}, \tilde{s}_{1}\right), y^{N}\right) \in \mathcal{T}_{\epsilon}^{N}$. The user index is guessed as $\hat{w}$ and the secret key is estimated as $\hat{s}_{1}$. If there is no such index triple or more than one index triple, an error is declared.

2) New System: For fixed $\delta$, given the rates in (6), pick a rate $R_{S}^{2}$ and an auxiliary rate pair $\left(\tilde{R}_{S}^{2}, R_{J}^{2}\right)$ such that

$$
\begin{aligned}
& \tilde{R}_{S}^{2}=I\left(U_{2} ; Y \mid U_{1}\right)-2 \delta, \\
& R_{S}^{2}=I\left(U_{2} ; Y \mid U_{1}\right)-2 \delta+\tilde{R}_{S}^{1}, \\
& R_{J}^{2}=I\left(U_{2} ; X \mid U_{1}\right)-I\left(U_{2} ; Y \mid U_{1}\right)+3 \delta .
\end{aligned}
$$

Codebook Generation: To construct the codebook of the new identification system, the codebook of the legacy system is also used. For each $u_{1}^{N}\left(j_{1}, s_{1}, \tilde{s}_{1}\right)$, randomly generate $2^{N\left(I\left(U_{2} ; X \mid U_{1}\right)+\delta\right)}$ i.i.d. codewords $u_{2}^{N}\left(j_{1}, s_{1}, \tilde{s}_{1}, j_{2}, \tilde{s}_{2}\right)$ according to $\prod_{i=1}^{N} P_{U_{2} \mid U_{1}=u_{1, i}\left(j_{1}, s_{1}, \tilde{s}_{1}\right)}$. We distribute the $2^{N\left(I\left(U_{2} ; X \mid U_{1}\right)+\delta\right)}$ codewords uniformly at random into $2^{N R_{J}^{2}}$ bins indexed by $j_{2}$, and each bin consists of $2^{N \tilde{R}_{S}^{2}}$ codewords, which are indexed by $\tilde{s}_{2}$. The codebooks and mappings are revealed to all parties.

Enrollment: For each user $w \in\left[1: 2^{N R_{I}}\right]$, do the procedure as in the legacy system to look for a codeword $u_{1}^{N}\left(j_{1}(w), s_{1}(w), \tilde{s}_{1}(w)\right)$. Then we look for a codeword $u_{2}^{N}\left(j_{1}(w), s_{1}(w), \tilde{s}_{1}(w), j_{2}(w), \tilde{s}_{2}(w)\right)$ such that

$$
\begin{aligned}
& \left(x^{N}(w), u_{1}^{N}\left(j_{1}(w), s_{1}(w), \tilde{s}_{1}(w)\right),\right. \\
& \left.u_{2}^{N}\left(j_{1}(w), s_{1}(w), \tilde{s}_{1}(w), j_{2}(w), \tilde{s}_{2}(w)\right)\right) \in \mathcal{T}_{\epsilon}^{N} .
\end{aligned}
$$

If no such $\left(j_{2}(w), \tilde{s}_{2}(w)\right)$ exists, an index pair $\left(j_{2}(w), \tilde{s}_{2}(w)\right)$ is randomly drawn from $\left[1: 2^{N R_{J}^{2}}\right] \times\left[1: 2^{N \tilde{R}_{S}^{2}}\right]$. If there are more than one such index pair $\left(j_{2}(w), \tilde{s}_{2}(w)\right)$, one of them is selected uniformly at random. The helper data and the secret key of user $w$ in the new system are generated as $j_{2}(w)$ and $s_{2}(w)=\left(\tilde{s}_{1}(w), \tilde{s}_{2}(w)\right)$, respectively.

Identification and Authentication: After user $w$ is observed, the observation $y^{N}$ is provided to the system. Firstly, we do the procedure as in the legacy system to look for a triple $\left(\hat{w}, \hat{s}_{1}, \tilde{s}_{1}, \tilde{s}_{2}\right)$ and the codeword $u_{1}^{N}\left(j_{1}(\hat{w}), \hat{s}_{1}, \tilde{s}_{1}\right)$. Then we look for a index $\tilde{s}_{2}$ such that $\left(u_{1}^{N}\left(j_{1}(\hat{w}), \hat{s}_{1}, \tilde{s}_{1}\right), u_{2}^{N}\left(j_{1}(\hat{w}), \hat{s}_{1}, \tilde{s}_{2}, j_{2}(\hat{w}), \tilde{s}_{2}\right), y^{N}\right) \in \mathcal{T}_{\epsilon}^{N}$. If there is no such index or more than one index, an error is declared.

Error Events Analysis: Assume that user $W$ is observed. Let $\left(J_{1}(W), S_{1}(W)\right)$ and $\left(J_{2}(W), S_{2}(W)=\right.$ $\left.\left(\tilde{S}_{1}(W), \tilde{S}_{2}(W)\right)\right)$ be the actual helper data and secret key of user $W$ for the legacy system and the new system, respectively. Let $\hat{W}, \hat{S}_{1}$ and $\hat{S}_{2}=\left(\widetilde{S}_{1}, \tilde{S}_{2}\right)$ denote the estimated user index and the secret keys for the legacy system and the new system, respectively.

Define event $\mathcal{E}_{1}=\left\{\left(\hat{W}, \hat{S}_{1}, \tilde{S}_{1}\right) \neq\left(W, S_{1}(W), \tilde{S}_{1}(W)\right)\right\}$. Following similar analysis as in [2, Appendix A-B], we can obtain that

$$
\operatorname{Pr}\left\{\mathcal{E}_{1}\right\} \leq \delta
$$

In the following, we condition on $\left(W, S_{1}(W), \tilde{S}_{1}(W)\right)$ being estimated correctly and provide the proof that $\tilde{S}_{2}(W)$ can be estimated reliably. Define the following event:

$$
\begin{aligned}
& A_{2}\left(\tilde{s}_{2}\right)=\left\{\left(Y^{N}, U_{1}^{N}\left(J_{1}(W), S_{1}(W), \tilde{S}_{1}(W)\right),\right.\right. \\
& \left.\left.\quad U_{2}^{N}\left(J_{1}(W), S_{1}(W), \tilde{S}_{1}(W), J_{2}(W), \tilde{s}_{2}\right)\right) \in \mathcal{T}_{\epsilon}^{N}\right\} .
\end{aligned}
$$

Then we have the following error events in the new system:

$$
\begin{aligned}
\mathcal{E}_{21}= & \left\{\left(U_{1}^{N}\left(J_{1}(W), S_{1}(W), \tilde{S}_{1}(W)\right),\right.\right. \\
& \left.U_{2}^{N}\left(J_{1}(W), S_{1}(W), \tilde{S}_{1}(W), j_{2}, \tilde{s}_{2}\right), X^{N}(W)\right) \notin \mathcal{T}_{\epsilon}^{N}, \\
& \left.\forall\left(j_{2}, \tilde{s}_{2}\right) \in\left[1: 2^{N R_{J}^{2}}\right] \times\left[1: 2^{N, \tilde{R}_{S}^{2}}\right]\right\}, \\
\mathcal{E}_{22}= & \left\{\left(Y^{N}, U_{1}^{N}\left(J(W), S_{1}(W), \tilde{S}_{1}(W)\right), U_{2}^{N}\left(J_{1}(W),\right.\right.\right. \\
& \left.\left.S_{1}(W), \tilde{S}_{1}(W), J_{2}(W), \tilde{S}_{2}(W)\right) \notin \mathcal{T}_{\epsilon}^{N}\right\} \\
\mathcal{E}_{23}= & \bigcup_{\tilde{s}_{2} \neq \tilde{S}_{2}(W)} A_{2}\left(\tilde{s}_{2}\right) .
\end{aligned}
$$

As for the probability of the error event $\mathcal{E}_{21}$, since $R_{J}^{2}+$ $\tilde{R}_{S}^{2}>I\left(U_{2} ; X \mid U_{1}\right)$, we obtain that $\operatorname{Pr}\left(\mathcal{E}_{21} \mid \mathcal{E}_{1}^{c}\right) \rightarrow 0$ as $N \rightarrow$ $\infty$ due to the covering lemma [8. Lemma 3.3].

The event $\left\{X^{N}(W)=X^{N}, U_{1}^{N}=u_{1}^{N}, U_{2}^{N}=u_{2}^{N}\right\}$ implies $Y^{N} \sim \prod_{i=1}^{N} P_{Y \mid X}\left(\cdot \mid \tilde{x}_{i}\right)$. By the Markov lemma [8, p.27], we obtain that $\operatorname{Pr}\left(\mathcal{E}_{22} \mid \mathcal{E}_{1}^{c}\right) \rightarrow 0$ as $N \rightarrow \infty$.

In the following error event analysis, due to symmetry and without loss of generality, we condition on the event $B=$ $\left\{W=1,\left(J_{1}(1), S_{1}(1), \tilde{S}_{1}(1), J_{2}(1), \tilde{S}_{2}(1)\right)=(1,1,1,1,1)\right\}$. The probability $\operatorname{Pr}\left\{\mathcal{E}_{23} \mid B\right\}$ can be bounded as follows

$$
\begin{aligned}
\operatorname{Pr}\left\{\mathcal{E}_{23} \mid B\right\} & \stackrel{(a)}{\leq} \sum_{\tilde{s}_{2} \neq 1} \operatorname{Pr}\left\{\left(U_{2}^{N}\left(1,1,1,1, \tilde{s}_{2}\right), Y^{N}\right) \in \mathcal{T}_{\epsilon}^{N} \mid B\right\} \\
& \stackrel{(b)}{\leq} 2^{N \tilde{R}_{S}^{2}}\left(1+\epsilon^{\prime}\right) 2^{-N\left(I\left(U_{2} ; Y \mid U_{1}\right)-\delta\right)}
\end{aligned}
$$

where $\epsilon^{\prime}>0$ is a fixed number; (a) holds due to the union bound; (b) follows from Lemma 1 in [9]. Since $\tilde{R}_{S}^{2} \leq$ $I\left(U_{2} ; Y \mid U_{1}\right)-\delta$, we obtain that $\operatorname{Pr}\left\{\mathcal{E}_{23} \mid B\right\} \rightarrow 0$ as $N \rightarrow \infty$. Consequently, using the union bound, we obtain that

$$
\operatorname{Pr}\left\{\mathcal{E}_{21} \cup \mathcal{E}_{22} \cup \mathcal{E}_{23} \mid \mathcal{E}_{1}^{c}\right\} \leq \delta .
$$

Combining (9) and (13), we obtain that

$$
\operatorname{Pr}\left\{\left(\hat{W}, \hat{S}_{i}\right) \neq\left(W, S_{i}(W)\right)\right\} \leq \delta, \quad j \in\{1,2\} .
$$

Therefore, we can conclude that there exists a suitable codebook $\mathcal{C}=C$ such that 3 a holds.

Secrecy Analysis: We firstly show that the codeword index tuple $\left(J_{1}(W), S_{1}(W), \tilde{S}_{1}(W), J_{2}(W), \tilde{S}_{2}(W)\right)$ is close to uniformly distributed. Due to symmetry, it is sufficient to consider $W=1$. For any $u_{1}^{N}\left(j_{1}, s_{1}, \tilde{s}_{1}\right)$ and $u_{2}^{N}\left(j_{1}, s_{1}, \tilde{s}_{1}, j_{2}, \tilde{s}_{2}\right)$, where $j_{1} \in\left[1: 2^{N R_{J}^{1}}\right], s_{1} \in\left[1: 2^{N R_{S}^{1}}\right], \tilde{s}_{1} \in\left[1: 2^{N \tilde{R}_{S}^{1}}\right]$, $j_{2} \in\left[1: 2^{N R_{J}^{2}}\right]$, and $\tilde{s}_{2} \in\left[1: 2^{N \tilde{R}_{S}^{2}}\right]$ we have

$$
\begin{aligned}
& \operatorname{Pr}\left\{X^{N}(1) \in \mathcal{T}_{\epsilon}^{N}\left(X \mid u_{1}^{N}\left(j_{1}, s_{1}, \tilde{s}_{1}\right), u_{2}^{N}\left(j_{1}, s_{1}, \tilde{s}_{1}, j_{2}, \tilde{s}_{2}\right)\right)\right\} \\
& \leq 2^{-N\left(I\left(U_{1}, U_{2} ; X\right)-\delta\right)}=2^{-N\left(I\left(U_{2} ; X\right)-\delta\right)} .
\end{aligned}
$$

Let $E$ be a random variable such that $E=0$ when there exists a codeword index tuple $\left(j_{1}, s_{1}, \tilde{s}_{1}, j_{2}, \tilde{s}_{2}\right)$ for user $W=$ 1 satisfying $\left(X^{N}(1), u_{1}^{N}\left(j_{1}, s_{1}, \tilde{s}_{1}\right), u_{2}^{N}\left(j_{1}, s_{1}, \tilde{s}_{1}, j_{2}, \tilde{s}_{2}\right)\right) \in$ $\mathcal{T}_{\epsilon}^{N}$. If no such codeword index exists, let $E=1$. Let $\gamma$ 
denote $\operatorname{Pr}(E=1)$. According to the error events analysis, we obtain that $\gamma \rightarrow 0$ as $N \rightarrow \infty$. Consider the joint entropy

$$
\begin{aligned}
& H\left(J_{1}(1), S_{1}(1), \tilde{S}_{1}(1), J_{2}(1), \tilde{S}_{2}(1)\right) \\
& =H\left(J_{1}(1), S_{1}(1), \tilde{S}_{1}(1), J_{2}(1), \tilde{S}_{2}(1), E\right) \\
& -H\left(E \mid J(1), S(1), \tilde{S}_{1}(1), J_{2}(1), \tilde{S}_{2}(1)\right) \\
& \geq H\left(J(1), S(1), \tilde{S}_{1}(1), J_{2}(1), \tilde{S}_{2}(1), E\right)-H(E) \\
& \stackrel{(a)}{\geq}-\sum_{j_{1}} \sum_{s_{1}} \sum_{\tilde{s}_{1}} \sum_{j_{2}} \sum_{\tilde{s}_{2}}\left[\operatorname { l o g } \left(\operatorname { P r } \left\{X^{N}(1) \in \mathcal{T}_{\epsilon}^{N}(X\right.\right.\right. \\
& \left.\left.\left.\mid u_{1}^{N}\left(j_{1}, s_{1}, \tilde{s}_{1}\right), u_{2}^{N}\left(j_{1}, s_{1}, \tilde{s}_{1}, j_{2}, \tilde{s}_{2}\right)\right)\right\}\right) \\
& \times \operatorname{Pr}\left\{J_{1}(1)=j_{1}, S_{1}(1)=s_{1}, \tilde{S}_{1}(1)=\tilde{s}_{1},\right. \\
& \left.\left.J_{2}(1)=j_{2}, \tilde{S}_{2}(1)=\tilde{s}_{2}, E=0\right\}\right]-h_{2}(\gamma) \\
& \geq \sum_{j_{1}} \sum_{s_{1}} \sum_{\tilde{s}_{1}} \sum_{j_{2}} \sum_{\tilde{s}_{2}} \operatorname{Pr}\left\{J_{1}(1)=j_{1}, S_{1}(1)=s_{1},\right. \\
& \left.\tilde{S}_{1}(1)=\tilde{s}_{1}, J_{2}(1)=j_{2}, \tilde{S}_{2}(1)=\tilde{s}_{2}, E=0\right\} \\
& \times N\left(I\left(U_{2} ; X\right)-\delta\right)-h_{2}(\gamma) \\
& =N\left(I\left(U_{2} ; X\right)-\delta\right)(1-\gamma)-h_{2}(\gamma) \text {, }
\end{aligned}
$$

where $h_{2}(\cdot)$ denote the binary entropy function, i.e., $h_{2}(p)=-p \log (p)-(1-p) \log (1-p)$; (a) holds because if $\left(J_{1}(1)=j_{1}, S_{1}(1)=s_{1}, \tilde{S}_{1}(1)=\tilde{s}_{1}, J_{2}(1)=\right.$ $\left.j_{2}, \tilde{S}_{2}(1)=\tilde{s}_{2}\right)$ and $E=0$ hold, then $X^{N}(1) \in$ $\mathcal{T}_{\epsilon}^{N}\left(X \mid u_{1}^{N}\left(j_{1}, s_{1}, \tilde{s}_{1}\right), u_{2}^{N}\left(j_{1}, s_{1}, \tilde{s}_{1}, j_{2}, \tilde{s}_{2}\right)\right)$ is valid. Therefore, $\quad\left(J_{1}(W), S_{1}(W), \widetilde{S}_{1}(W), J_{2}(W), \tilde{S}_{2}(W)\right)$ is close to uniformly distributed. Consequently, $S_{1}(W), S_{2}(W)$, and $\left(S_{1}(W), S_{2}(W)\right)$ are also close to uniformly distributed, i.e.,

$$
\begin{aligned}
H\left(S_{1}(W)\right) & \geq N\left(R_{S}^{1}-\xi\right), \\
H\left(S_{2}(W)\right) & \geq N\left(R_{S}^{2}-\xi\right), \\
H\left(S_{1}(W), S_{2}(W)\right) & \geq N\left(R_{S}^{1}+R_{S}^{2}-\xi\right),
\end{aligned}
$$

where $\xi \rightarrow 0$ as $N \rightarrow \infty$. Hence we have that

$$
\begin{aligned}
& I\left(S_{1}(W) ; S_{2}(W)\right) \\
& =H\left(S_{1}(W)\right)+H\left(S_{2}(W)\right)-H\left(S_{1}(W), S_{2}(W)\right) \\
& \stackrel{(a)}{\leq} N R_{S}^{1}+N R_{S}^{2}-N\left(R_{S}^{1}+R_{S}^{2}-\xi\right)=N \xi
\end{aligned}
$$

where (a) follows from the fact that $S_{i}(W) \in\left[1: 2^{N R_{S}^{i}}\right]$ for $i \in\{1,2\}$, and thus $H\left(S_{i}(W)\right) \leq N R_{S}^{i}$ for $i \in\{1,2\}$. Therefore, the requirement in (3f) is satisfied.

We obtain for the secrecy leakage

$$
\begin{aligned}
& I\left(S_{1}(W), S_{2}(W) ; J_{1}(W), J_{2}(W)\right) \\
& =H\left(S_{1}(W), S_{2}(W)\right)+H\left(J_{1}(W), J_{2}(W)\right) \\
& \left.\quad-H\left(J_{1}(W), J_{2}(W), S_{1}(W), S_{2}(W)\right)\right) \\
& \stackrel{(a)}{\leq} N\left(I\left(U_{2} ; X\right)+2 \delta\right)-\left[N\left(I\left(U_{2} ; X\right)-\delta\right)(1-\gamma)-h_{2}(\gamma)\right] \\
& =N \delta^{\prime}
\end{aligned}
$$

where $\delta^{\prime}=2 \delta+\gamma(I(U ; X)-\delta)+\frac{1}{N} h_{2}(\gamma)$ and $\delta^{\prime} \rightarrow 0$ as $N \rightarrow \infty$; (a) follows from (6), (7), and (16). Therefore, the secrecy requirement in $3 \mathrm{e}$ is satisfied.
Privacy Analysis: We obtain for the privacy leakage

$$
\begin{aligned}
& I\left(X^{N}(W) ; J_{1}(W), J_{2}(W)\right) \leq H\left(J_{1}(W), J_{2}(W)\right) \\
& \quad \leq H\left(J_{1}(W)\right)+H\left(J_{2}(W)\right) \leq R_{J}^{1}+R_{J}^{2} \\
& \quad=N\left(I\left(U_{2} ; X \mid Y\right)+R_{I}+6 \delta\right) .
\end{aligned}
$$

Combining (6), (7), (14), (17), (18), (20), (21), and (22), we complete the achievability proof of Theorem 1

\section{B. Converse}

Fix $\delta>0$ and for this $\delta$ there exists a codebook with rate tuple $\left(R_{I}, R_{L}, R_{S}^{1}, R_{S}^{2}\right)$ satisfying the conditions in (3). For such code, Fano's inequality implies that $H\left(W, S_{1}(W), S_{2}(W) \mid \hat{W}, \hat{S}_{1}, \hat{S}_{2}\right) \leq F$, where $F \triangleq$ $1+\operatorname{Pr}\left\{\left(\hat{W}, \hat{S}_{1}, \hat{S}_{2}\right) \neq\left(W, S_{1}(W), S_{2}(W)\right)\right\} \log \left(M_{I} M_{S}^{1} M_{S}^{2}\right)$. Similarly, it holds that $H\left(W, S_{1}(W) \mid \hat{W}, \hat{S}_{1}\right) \leq F_{1}$, where $F_{1} \triangleq 1+\operatorname{Pr}\left\{\left(\hat{W}, \hat{S}_{1}\right) \neq\left(W, S_{1}(W)\right)\right\} \log \left(M_{I} M_{S}^{1}\right)$. Therefore $\frac{F}{N} \rightarrow 0$ and $\frac{F_{1}}{N} \rightarrow 0$ as $\operatorname{Pr}\left\{\left(\hat{W}, \hat{S}_{i}\right) \neq\left(W, S_{i}(W)\right)\right\} \rightarrow 0$ and $N \rightarrow \infty$ for $i \in\{1,2\}$. We define auxiliary random variables $U_{1, n}=\left(W, J_{1}(W), S_{1}(W), X^{n-1}(W)\right)$ and $U_{2, n}=$ $\left(U_{1, n}, J_{2}(W), S_{2}(W)\right)$ for $n \in[1: N]$.

To defer the proof, we include the following claims.

Claim 1: It can be seen that

$$
\begin{aligned}
& H\left(W, S_{1}(W), S_{2}(W) \mid \boldsymbol{J}_{1}, \boldsymbol{J}_{2}, Y^{N}\right) \\
& \quad \stackrel{(a)}{\leq} H\left(W, S_{1}(W), S_{2}(W) \mid \hat{W}, \hat{S}_{1}, \hat{S}_{2}\right) \stackrel{(b)}{\leq} F,
\end{aligned}
$$

where (a) follows from that $\left(\hat{W}, \hat{S}_{1}, \hat{S}_{2}\right)$ is a function of $\left(\boldsymbol{J}_{1}, \boldsymbol{J}_{2}, Y^{N}\right)$; (b) follows from Fano's inequality.

Claim 2: It can be seen that

$$
\begin{aligned}
& I\left(W, S_{1}(W), S_{2}(W), \boldsymbol{J}_{1}, \boldsymbol{J}_{2} ; Y^{N}\right) \\
& \stackrel{(a)}{=} I\left(W, S_{1}(W), S_{2}(W), J_{1}(W), J_{2}(W) ; Y^{N}\right) \\
& \stackrel{(b)}{\leq} \sum_{n=1}^{N} I\left(Y_{n} ; U_{2, n}\right),
\end{aligned}
$$

where (a) follows from the fact that given the user index $W$, $Y^{N}$ is independent of the helper data of the other users; (b) follows from the Markov chain $Y^{n-1}-U_{2, n}-Y_{n}$.

Now we consider the following entropy

$$
\begin{aligned}
& H\left(W, S_{1}(W), S_{2}(W)\right) \\
& =I\left(W, S_{1}(W), S_{2}(W) ; \boldsymbol{J}_{1}, \boldsymbol{J}_{2}, Y^{N}\right) \\
& \quad+H\left(W, S_{1}(W), S_{2}(W) \mid \boldsymbol{J}_{1}, \boldsymbol{J}_{2}, Y^{N}\right) \\
& \quad \stackrel{(a)}{\leq} I\left(W, S_{1}(W), S_{2}(W) ; \boldsymbol{J}_{1}, \boldsymbol{J}_{2}\right) \\
& \quad+I\left(W, S_{1}(W), S_{2}(W) ; Y^{N} \mid \boldsymbol{J}_{1}, \boldsymbol{J}_{2}\right)+F \\
& \quad \stackrel{(b)}{\leq} I\left(W ; \boldsymbol{J}_{1}, \boldsymbol{J}_{2}\right)+I\left(S_{1}(W), S_{2}(W) ; J_{1}(W), J_{2}(W)\right) \\
& \quad+I\left(W, S_{1}(W), S_{2}(W), \boldsymbol{J}_{1}, \boldsymbol{J}_{2} ; Y^{N}\right)+F \\
& \quad \text { (c) } \\
& \leq N \delta+\sum_{n=1}^{N} I\left(Y_{n} ; U_{2, n}\right)+F,
\end{aligned}
$$

where (a) follows from Claim 1; (b)holds because given $W$, $\left(S_{1}(W), S_{2}(W)\right)$ is independent of the helper data of other users; (c) follows from (3e) and Claim 2. Then we obtain that $\log M_{I} M_{S}^{1} M_{S}^{2} \stackrel{(a)}{\leq} N\left(H(W)+H\left(S_{1}(W), S_{2}(W)\right)\right)+3 N \delta$ 


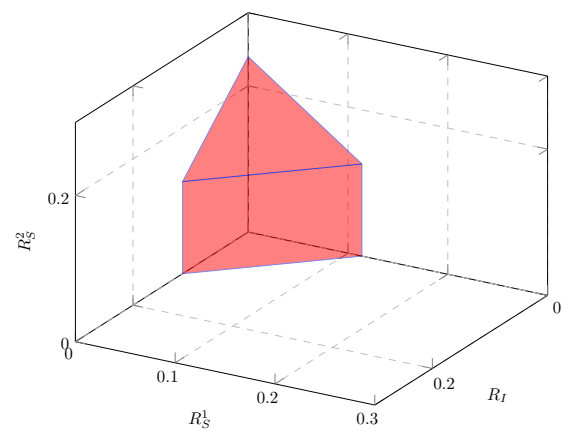

(a) Trade-offs among $\left(R_{I}, R_{S}^{1}, R_{S}^{2}\right)$.

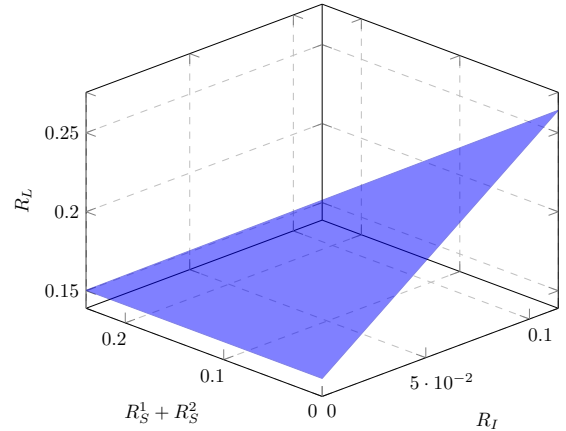

(b) Trade-offs among $\left(R_{I}, R_{S}^{1}+R_{S}^{2}, R_{L}\right)$.

Fig. 2: Trade-offs among $\left(R_{I}, R_{S}^{1}, R_{S}^{2}, R_{L}\right)$ when $q=0.1$ and $p_{1}=p_{2}=0.15$.

$$
\begin{aligned}
& \stackrel{(b)}{=} N H\left(W, S_{1}(W), S_{2}(W)\right)+3 N \delta \\
& \stackrel{(c)}{\leq} \sum_{n=1}^{N} I\left(Y_{n} ; U_{2, n}\right)+F+4 N \delta,
\end{aligned}
$$

where (a) follows from $(3 \mathrm{c})$ and $(3 \mathrm{f})$; (b) follows from the fact that the user index $W$ is independent of secret keys $S_{1}(W)$ and $S_{2}(W)$; (c) follows from 25 ).

Combining (26) with (3b) and (3c), we obtain that

$$
\begin{aligned}
R_{I}+R_{S}^{1}+R_{S}^{2} & \leq \frac{\log M_{I} M_{S}^{1} M_{S}^{2}}{N}+2 \delta \\
& \leq \frac{1}{N} \sum_{n=1}^{N} I\left(Y_{n} ; U_{2, n}\right)+\frac{F}{N}+6 \delta .
\end{aligned}
$$

Following similar analysis in (25), 26), and (27), we obtain that

$$
R_{I}+R_{S}^{1} \leq \frac{1}{N} \sum_{n=1}^{N} I\left(Y_{n} ; U_{1, n}\right)+\frac{F_{1}}{N}+3 \delta
$$

The privacy leakage rate can be bounded as follow

$$
\begin{aligned}
& N\left(R_{L}+\delta\right) \geq I\left(X^{N}(W) ; J_{1}(W), J_{2}(W)\right) \\
& \stackrel{(a)}{=} I\left(X^{N}(W) ; \boldsymbol{J}_{1}, \boldsymbol{J}_{2} \mid W\right)=I\left(X^{N}(W), W ; \boldsymbol{J}_{1}, \boldsymbol{J}_{2}\right) \\
& =H\left(X^{N}(W)\right)-H\left(X^{N}(W), W, S_{1}(W), S_{2}(W) \mid \boldsymbol{J}_{1}, \boldsymbol{J}_{2}\right) \\
& \quad+H(W)+H\left(S_{1}(W), S_{2}(W) \mid \boldsymbol{J}_{1}, \boldsymbol{J}_{2}, X^{N}(W), W\right) \\
& =I\left(X^{N}(W) ; W, S_{1}(W), S_{2}(W), \boldsymbol{J}_{1}, \boldsymbol{J}_{2}\right) \\
& \quad+N R_{I}-H\left(W, S_{1}(W), S_{2}(W) \mid X^{N}(W), \boldsymbol{J}_{1}, \boldsymbol{J}_{2}\right) \\
& \stackrel{(b)}{\geq} I\left(X^{N}(W) ; W, S_{1}(W), S_{2}(W), J_{1}(W), J_{2}(W)\right) \\
& \quad+N R_{I}-I\left(W, S_{1}(W), S_{2}(W) ; Y^{N} \mid \boldsymbol{J}_{1}, \boldsymbol{J}_{2}\right) \\
& \quad-H\left(W, S_{1}(W), S_{2}(W) \mid Y^{N}, \boldsymbol{J}_{1}, \boldsymbol{J}_{2}\right) \\
& \quad \stackrel{(c)}{\geq} I\left(X^{N}(W) ; W, S_{1}(W), S_{2}(W), J_{1}(W), J_{2}(W)\right) \\
& \quad+N R_{I}-I\left(W, S_{1}(W), S_{2}(W), \boldsymbol{J}_{1}, \boldsymbol{J}_{2} ; Y^{N}\right)-F \\
& \stackrel{(d)}{\geq} \sum_{i=1}^{N}\left(I\left(X_{n}(W) ; U_{2, n}\right)-I\left(Y_{n} ; U_{2, n}\right)\right)-F+N R_{I} \\
& \quad \stackrel{(e)}{\geq} \sum_{i=1}^{N} I\left(X X_{n}(W) ; U_{2, n} \mid Y_{n}\right)-F+N R_{I},
\end{aligned}
$$

where (a) holds since given $W, X^{N}(W)$ is independent of the helper data of the other users; (b) holds since conditioning reduces entropy; (c) follows from Claim 1. (d) follows from Claim 2, (e) follows from $U_{2, n}-X_{n}(W)-Y_{n}$.

Combining (27), 28), and (29), we complete the converse proof.

\section{BINARY EXAMPLE}

Suppose the source $X$ is binary and uniformly distributed. Assume the observation channel $Q_{Y \mid X}$ is a binary symmetric channel (BSC) with crossover probability $q$. We choose $U_{2}$ to be an output of a BSC with crossover probability $p_{1}$ and input $X$, and choose $U_{1}$ to be an output of a BSC with crossover probability $p_{2}$ and input $U_{2}$. According to Mrs. Gerber's lemma [10], we obtain that if $H\left(Y \mid U_{2}\right) \geq h_{2}\left(p_{1} * q\right)$ for some $p_{1}, q \in\left(0, \frac{1}{2}\right)$, where $*$ denotes the operation of $p * q=p(1-q)+q(1-p)$. Similarly, we obtain that $H\left(Y \mid U_{1}\right) \geq h_{2}\left(p_{1} * p_{2} * q\right)$, and $H\left(X \mid U_{1}\right) \geq h_{2}\left(p_{1} * p_{2}\right)$. The achievable region of this problem becomes

$$
\begin{aligned}
& R_{I}+R_{S}^{1} \leq 1-h_{2}\left(p_{1} * p_{2} * q\right), \\
& R_{I}+R_{S}^{1}+R_{S}^{2} \leq 1-h_{2}\left(p_{1} * q\right), \\
& R_{L} \leq h_{2}\left(p_{1} * q\right)-h_{2}\left(p_{1}\right)+R_{I} .
\end{aligned}
$$

Here we choose $p_{1}=p_{2}=0.15$ and $q=0.1$. The tradeoffs boundaries among $\left(R_{I}, R_{S}^{1}, R_{S}^{2}, R_{L}\right)$ are depicted in Fig. 2. Any rate tuples with a smaller identification rate, smaller secret key rates, or a larger privacy leakage rate than the rate tuples on the boundaries are achievable. Observe Fig. 2a, we can see that an increase of one of the three rates $\left(R_{I}, R_{S}^{1}, R_{S}^{2}\right)$ results in a decrease in the other two rates. Similar observation can also be seen for rate pair $\left(R_{I}, R_{S}^{1}\right)$ in Fig. (2a), i.e., by fixing $R_{S}^{2}$, the increase of $R_{I}$ results in the decrease of $R_{S}^{1}$ and vise versa. This is because the public database is used for both identification and authentication, i.e., guessing the user index and estimating the secret key. Hence if more users are enrolled, the secret key rate would be sacrificed. Observe Fig. 2b, we see that by fixing $R_{S}^{1}+R_{S}^{2}, R_{L}$ increases as $R_{I}$ increases. However, by fixing $R_{I}, R_{L}$ does not change by increasing or decreasing $R_{S}^{1}+R_{S}^{2}$. This can be interpreted as that the secret keys are stored in secure databases and thus information can not be leaked from the secret keys. Hence the privacy leakage rate does not directly depend on the secret key rate. 


\section{REFERENCES}

[1] T. Ignatenko and F. M. J. Willems, "Biometric systems: Privacy and secrecy aspects," IEEE Tran. Inf. Forensics Security, vol. 4, no. 4, pp. 956-973, Dec. 2009.

[2] — - "Fundamental limits for privacy-preserving biometric identification systems that support authentication," IEEE Trans. Inf. Theory, vol. 61 , no. 10 , pp. 5583-5594, Oct 2015.

[3] F. Willems, T. Kalker, J. Goseling, and J. P. Linnartz, "On the capacity of a biometrical identification system," in Proc. IEEE Int. Symp. Inf. Theory, Jun. 2003, pp. 82-87.

[4] E. Tuncel, "Capacity/storage tradeoff in high-dimensional identification systems," in Proc. IEEE Int. Symp. Inf. Theory, Jul. 2006, pp. 19291933.

[5] K. Kittichokechai and G. Caire, "Secret key-based identification and authentication with a privacy constraint," IEEE Trans. Inf. Theory, vol. 62, no. 11, pp. 6189-6203, 2016.

[6] L. Lai, S. Ho, and H. V. Poor, "Privacy-security trade-offs in biometric security systems-part ii: Multiple use case," IEEE Trans. Inf. Forensics Security, vol. 6, no. 1, pp. 140-151, March 2011.

[7] W. H. Equitz and T. M. Cover, "Successive refinement of information," IEEE Trans. Inf. Theory, vol. 37, no. 2, pp. 269-275, 1991.

[8] A. El Gamal and Y.-H. Kim, Network information theory. Cambridge university press, 2011.

[9] M. T. Vu, T. J. Oechtering, M. Wiese, and M. Skoglund, "Cooperative successive refinement with side information," manuscript in preparation. [Online]. Available: https://people.kth.se/ oech/jnlp.pdf

[10] A. Wyner, "A theorem on the entropy of certain binary sequences and applications-i," IEEE Trans. Inf. Theory, vol. 19, no. 6, pp. 769-772, 1973. 